\title{
A Remark on Quantum Group Actions and Nuclearity
}

\author{
S. DOPLICHER \\ Dipartimento di Matematica \\ Università di Roma "La Sapienza" \\ Piazzale A. Moro 5, I-00185 Roma, Italy \\ R. Longo, J.E. Roberts, L. Zsidó \\ Dipartimento di Matematica \\ Università di Roma "Tor Vergata" \\ Via della Ricerca Scientifica 1, I-00133 Roma, Italy \\ Dedicated to Huzihiro Araki \\ on the occasion of his seventieth birthday
}

\begin{abstract}
Let $H$ be a compact quantum group with faithful Haar measure and bounded counit. If $\alpha$ is an action of $H$ on a $C^{*}$-algebra $A$, we show that $A$ is nuclear if and only if the fixed-point subalgebra $A^{\alpha}$ is nuclear. As a consequence $H$ is a nuclear $C^{*}$-algebra.
\end{abstract}

Supported in part by MIUR and GNAMPA-INDAM.

E-mails: dopliche@mat.uniroma1.it, longo@mat.uniroma2.it,roberts@mat.uniroma2.it, zsido@mat.uniroma2.it 


\section{Introduction}

The notion of compact quantum group has been axiomatized by Woronowicz [16] and some variations appeared in subsequent papers, e.g. [1, 12, 13, 9]. Here we shall consider compact quantum groups allowing a faithful Haar state and bounded counit, conditions that cover a rather wide range of applicability cf. 15.].

In this note we will show that a $C^{*}$-algebra acted upon by a compact quantum group with faithful Haar state and bounded counit is nuclear if and only if the fixed point algebra is nuclear. In particular, a compact quantum group with faithful Haar state and bounded counit is a nuclear $C^{*}$-algebra. Notice however that our assumptions about the faithfulness of the Haar state and the boundedness of the counit are essential: there exist non-nuclear compact quantum groups, as we can infer from [15], second remark after the statement of Proposition 1.8, and [13].

As a special case, a $C^{*}$-algebra acted upon by a compact group of automorphisms is nuclear if and only if the fixed point algebra is nuclear. Indeed this was our initial motivation, during a common discussion long ago at the Rome seminar on Operator Algebras, inspired by a result of Høegh-Krohn, Landstad and Størmer [8] showing that there is a compact ergodic action on a $C^{*}$-algebra only if the algebra is nuclear, and by a lemma in [6]. This particular case is discussed separately as it is a simple and clarifying illustration for the general case.

We notice that another extension of the result of Høegh-Krohn, Landstad and Størmer to compact matrix pseudogroups was already proved by Boca in [2], Corollary 23: any $C^{*}$-algebra acted upon ergodically by a nuclear compact matrix pseudogroup is again nuclear. Actually Boca's proof works in the case of an ergodic action of an arbitrary nuclear compact quantum group.

We also analyse compact actions on von Neumann algebras where the analogous result holds for injective von Neumann algebras. This $W^{*}$-version can however also be inferred from a similar result for crossed products due to Connes [5] and indeed can be extended in part to the case of integrable actions of locally compact groups or Kac algebras, but we do not treat this case here.

Our discussion is elementary, but in the last section $\left(W^{*}\right.$-case) we make use of the relation between injectivity and semi-discreteness due to EffrosLance, Choi-Effros and Connes [7, 3, 4]. 


\section{Notation:}

If $\mathcal{A}$ is a ${ }^{*}$-algebra we shall denote the identity map on $\mathcal{A}$ by $\iota_{\mathcal{A}}$, or simply by $\iota$. When $\mathcal{A}$ is unital, we shall denote its unit by $1_{\mathcal{A}}$.

The maximal tensor product for $C^{*}$-algebras is denoted by $\otimes_{\max }$, the minimal one by $\otimes_{\min }$ or simply by $\otimes$, the algebraic tensor product by $\odot$. For a $C^{*}$-algebra $A$, we shall usually identify $\mathbb{C} \otimes A$ and $A \otimes \mathbb{C}$ with $A$.

By a homomorphism we shall always mean a ${ }^{*}$-homomorphism.

\section{Basic Result}

Let us call a conditional expectation $\mathcal{E}$ on a $C^{*}$-algebra $A$ GNS-faithful if

$$
x \in A \& \mathcal{E}\left(y^{*} x^{*} x y\right)=0 \text { for } \forall y \in A \Longrightarrow x=0 .
$$

This means that the direct sum of the GNS representations associated with all $\mathcal{E}$-invariant states is injective. Of course, $\mathcal{E}$ is GNS-faithful if $\mathcal{E}$ is faithful.

Let $A$ be a $C^{*}$-algebra, $A_{0} \subset A$ a $C^{*}$-subalgebra and $\mathcal{E}: A \rightarrow A_{0}$ a conditional expectation.

If $B$ is any $C^{*}$-algebra, it is easy to see that $\iota_{B} \odot \mathcal{E}: B \odot A \rightarrow B \odot A_{0}$ extends to a conditional expectation from $B \otimes_{\min } A$ to $B \otimes_{\min } A_{0}$, which is (GNS-)faithful if $\mathcal{E}$ is (GNS-)faithful and which we denote by $\iota_{B} \otimes \mathcal{E}$.

We shall say that $\mathcal{E}$ is stably (GNS-)faithful if, for every $C^{*}$-algebra $B$, $\iota_{B} \odot \mathcal{E}$ extends to a bounded (GNS-)faithful map $\tilde{\mathcal{E}}: B \otimes_{\max } A \rightarrow B \otimes_{\max } A$. In this case $\tilde{\mathcal{E}}$ is a (GNS-)faithful conditional expectation from $B \otimes_{\max } A$ onto the closure of $B \odot A_{0}$ in $B \otimes_{\max } A$.

Proposition 1. Let $A$ be a $C^{*}$-algebra, $A_{0} \subset A$ a $C^{*}$-subalgebra and $\mathcal{E}$ : $A \rightarrow A_{0}$ a stably GNS-faithful conditional expectation. Then $A$ is nuclear iff $A_{0}$ is nuclear.

Proof. The implication $A$ nuclear $\Longrightarrow A_{0}$ nuclear is known (indeed one just needs $A_{0}$ to be the range of a conditional expectation). One way to see this is to recall that $A$ is nuclear iff the enveloping von Neumann algebra $A^{* *}$ is injective [7, 3], and to consider the double transposed conditional expectation $\mathcal{E}^{* *}: A^{* *} \rightarrow A_{0}^{* *}$. If $A$ is nuclear, $A^{* *}$ is injective, and then $A_{0}^{* *}$ is injective too, hence $A_{0}$ is nuclear.

Conversely, assume that $A_{0}$ is nuclear. Given a $C^{*}$-algebra $B$, the identity map on the algebraic tensor product $B \odot A$ extends to a homomorphism

$$
\pi: B \otimes_{\max } A \rightarrow B \otimes_{\min } A .
$$


To show that $A$ is nuclear, we have to prove $\pi$ to be one-to-one.

By assumption, there is a GNS-faithful conditional expectation $\tilde{\mathcal{E}}: B \otimes_{\max }$ $A \rightarrow B \otimes_{\max } A$ extending $\iota_{B} \odot \mathcal{E}$. Denoting the conditional expectation $\iota_{B} \otimes \mathcal{E}$ on $B \otimes_{\min } A$ by $\tilde{\mathcal{E}}^{\prime}$, we clearly have a commutative diagram

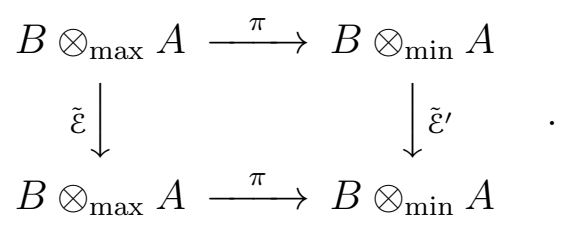

Since $\tilde{\mathcal{E}}$ maps $B \odot A$ onto $B \odot A_{0}$, by continuity $\tilde{\mathcal{E}}$ maps $B \otimes_{\max } A$ onto the closure of $B \odot A_{0}$ in $B \otimes_{\max } A$, that we may denote by $B \otimes A_{0}$ since $A_{0}$ is nuclear. Then (11) yields the commutative diagram

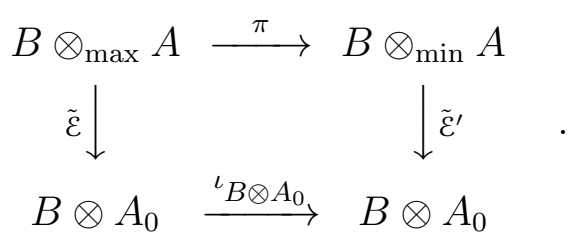

Now if $x \in B \otimes_{\max } A$ belongs to the kernel of $\pi$, then $\tilde{\mathcal{E}}^{\prime}\left(\pi\left(y^{*} x^{*} x y\right)\right)=$ 0 for all $y \in B \otimes_{\max } A$. By the commutativity of diagram (2) we have $\tilde{\mathcal{E}}\left(y^{*} x^{*} x y\right)=0$ for all $y \in B \otimes_{\max } A$, so $x=0$ because $\tilde{\mathcal{E}}$ is GNS-faithful. We conclude that $\pi$ is injective and $A$ nuclear.

\section{Compact Group Actions and Nuclearity}

In the sequel, group actions on $C^{*}$-algebras and von Neumann algebras will be assumed continuous in the usual sense, namely pointwise norm continuity in the $C^{*}$-case and pointwise weak ${ }^{*}$-continuity in the $W^{*}$-case.

Proposition 2. Let $\alpha: G \rightarrow \operatorname{Aut}(A)$ be an action of a compact group $G$ on a $C^{*}$-algebra $A$. Then $A$ is nuclear iff the fixed point $C^{*}$-subalgebra $A^{\alpha}$ is nuclear.

Proof. Let $\mathcal{E}_{\alpha}: A \rightarrow A^{\alpha}$ be the conditional expectation defined by

$$
\mathcal{E}_{\alpha}(a)=\int \alpha_{g}(a) \mathrm{d} g, \quad a \in A
$$


According to Proposition 1, it suffices to show that $\mathcal{E}_{\alpha}$ is stably faithful.

The action $\beta=\iota_{B} \otimes \alpha$ on $B \odot A$ preserves the maximal cross norm $\|\cdot\|_{\max }$. Furthermore, $G \ni g \mapsto \beta_{g}(x)$ is continuous for every $x \in B \odot A$ with respect to $\|\cdot\|_{\max }$. Thus $\beta$ extends to an action $\beta^{\max }$ of $G$ on $B \otimes_{\max } A$.

Set

$$
\mathcal{E}_{\beta}(x)=\int \beta_{g}^{\max }(x) \mathrm{d} g, \quad x \in B \otimes_{\max } A .
$$

Then $\mathcal{E}_{\beta}$ is a faithful conditional expectation because we have

$$
\mathcal{E}_{\beta}(x)=\int \beta_{g}^{\max }(x) \mathrm{d} g=0 \Longrightarrow \beta_{g}^{\max }(x)=0 \forall g \in G
$$

for every positive $x \in B \otimes_{\max } A$. Since $\mathcal{E}_{\beta}$ maps $B \odot A$ onto $B \odot A^{\alpha}$ and $\left.\mathcal{E}_{\beta}\right|_{B \odot A}=\iota \odot \mathcal{E}_{\alpha}$, we see that $\mathcal{E}_{\alpha}$ is stably faithful.

\section{Quantum Group Actions and Nuclearity}

Following Woronowicz and Van Daele [16, 12, by a compact quantum group we shall mean a unital $C^{*}$-algebra $H$ equipped with a comultiplication $\delta$, that is a unital homomorphism $\delta: H \rightarrow H \otimes H$ satisfying

$$
\left(\delta \otimes \iota_{H}\right) \circ \delta=\left(\iota_{H} \otimes \delta\right) \circ \delta,
$$

such that

$$
\delta(H)\left(H \otimes 1_{H}\right) \text { and } \delta(H)\left(1_{H} \otimes H\right) \text { are linearly dense in } H \otimes H .
$$

According to [16, 12], there then exists a unique Haar state on $H$, that is a state $\varphi$ which satisfies the invariance condition

$$
\left(\left(\varphi \otimes \iota_{H}\right) \circ \delta\right)(x)=\left(\left(\iota_{H} \otimes \varphi\right) \circ \delta\right)(x)=\varphi(x) 1_{H}, \quad x \in H .
$$

We notice that by condition (3) the fixed point algebra

$$
H^{\delta}=\left\{x \in H ; \delta(x)=x \otimes 1_{H}\right\} \text { is equal to } \mathbb{C} 1_{H} .
$$

The unique Haar state $\varphi$ is not necessarily faithful. However, the cyclic vector $\xi_{\varphi}$ of the GNS-representation $\pi_{\varphi}$ is also separating for $\pi_{\varphi}(H)^{\prime \prime}$. Indeed, $\delta$ lifts to a comultiplication $\delta_{\varphi}$ on $M_{\varphi}=\pi_{\varphi}(H)^{\prime \prime}$ (see e.g. [14, Theorem 
2.4) and the vector state $\omega_{\xi_{\varphi}}$ on $M_{\varphi}$ satisfies the invariance conditions corresponding to (3), in particular $M_{\varphi}^{\delta_{\varphi}}=\mathbb{C} 1_{M_{\varphi}}$. Now [1], Lemma 0.2.4, implies that the support of $\omega_{\xi_{\varphi}} \mid M_{\varphi}$ belongs to $M_{\varphi} \delta_{\varphi}=\mathbb{C} 1_{M_{\varphi}}$, hence it is equal to $1_{M_{\varphi}}$. Consequently, replacing $H$ with $H / \operatorname{ker}\left(\pi_{\varphi}\right)$ if necessary, one can consider the case where $\varphi$ is faithful, cf. e.g. [15], remarks after Theorem 5.6, [10, 14].

According to [16], Theorem 2.2, the linear $\operatorname{span} \mathcal{A}$ of all matrix elements of all finite-dimensional unitary representations of $H$ is a dense ${ }^{*}$-subalgebra of $H$ with $1_{H} \in \mathcal{A}, \delta(\mathcal{A}) \subset \mathcal{A} \odot \mathcal{A}$ and there are unique linear maps $\epsilon: \mathcal{A} \rightarrow \mathbb{C}$ and $\kappa: \mathcal{A} \rightarrow \mathcal{A}$, called respectively counit and coinverse or antipode, such that

$$
\begin{aligned}
\left(\epsilon \odot \iota_{\mathcal{A}}\right)(\delta(a)) & =\left(\iota_{\mathcal{A}} \odot \epsilon\right)(\delta(a))=a, & & a \in \mathcal{A}, \\
\left(m \circ\left(\kappa \odot \iota_{\mathcal{A}}\right)\right)(\delta(a)) & =\left(m \circ\left(\iota_{\mathcal{A}} \odot \kappa\right)\right)(\delta(a))=\epsilon(a) 1_{H}, & & a \in \mathcal{A},
\end{aligned}
$$

where the linear map $m: \mathcal{A} \odot \mathcal{A} \rightarrow \mathcal{A}$ is defined by $m(a \odot b)=a b, a, b \in \mathcal{A}$.

The counit $\epsilon$ is a multiplicative positive linear functional on $\mathcal{A}$ with $\epsilon\left(1_{H}\right)=1$, but it is in general not bounded. However, it is often bounded and then it extends to a multiplicative state on $H$, still denoted by $\epsilon$, which satisfies

$$
\left(\epsilon \otimes \iota_{H}\right) \circ \delta=\left(\iota_{H} \otimes \epsilon\right) \circ \delta=\iota_{H} .
$$

We notice that (5) implies the injectivity of $\delta$.

In the rest of this section $H$ will denote a compact quantum group with faithful Haar state and bounded counit. This is the case for compact groups and for the quantum $U(N)$-group, but not for the dual of a non-amenable discrete group, see [15], second remark after the statement of Proposition 1.8 .

By a coaction $\alpha$ of $H$ on a $C^{*}$-algebra $A$ we mean a homomorphism $\alpha: A \rightarrow A \otimes H$ such that

$$
\begin{aligned}
\left(\alpha \otimes \iota_{H}\right) \circ \alpha & =\left(\iota_{A} \otimes \delta\right) \circ \alpha \\
\left(\iota_{A} \otimes \epsilon\right) \circ \alpha & =\iota_{A} .
\end{aligned}
$$

The last equation implies that $\alpha$ is injective.

The fixed-point subalgebra is then defined by

$$
A^{\alpha}=\left\{a \in A ; \alpha(a)=a \otimes 1_{H}\right\} .
$$


Denoting

$$
\mathcal{E}=\left(\iota_{A} \otimes \varphi\right) \circ \alpha: A \rightarrow A,
$$

we have the known fact:

Lemma 3. $\mathcal{E}$ is a faithful conditional expectation from $A$ to $A^{\alpha}$.

Proof. $\mathcal{E}$ is a faithful map, being the composition of faithful maps. Clearly $A^{\alpha}$ is contained in the range of $\mathcal{E}$.

We now apply standard calculations and check that $\mathcal{E}$ is idempotent. Indeed, identifying $A$ with $A \otimes \mathbb{C}$, we get

$$
\begin{aligned}
\mathcal{E}^{2} & =\left(\left(\iota_{A} \otimes \varphi\right) \circ \alpha\right) \circ\left(\iota_{A} \otimes \varphi\right) \circ \alpha \\
& =\left(\iota_{A} \otimes \varphi\right) \circ(\alpha \otimes \varphi) \circ \alpha \\
& =\left(\iota_{A} \otimes \varphi \otimes \varphi\right) \circ\left(\alpha \otimes \iota_{H}\right) \circ \alpha \\
& =\left(\iota_{A} \otimes \varphi \otimes \varphi\right) \circ\left(\iota_{A} \otimes \delta\right) \circ \alpha \\
& =\left(\iota_{A} \otimes(\underbrace{(\varphi \otimes \varphi) \circ \delta}_{=\varphi(\cdot) \cdot \mathbb{1}_{\mathbb{C}} \otimes \mathbb{1}_{\mathbb{C}}})\right) \alpha \\
& =\left(\iota_{A} \otimes \varphi\right) \circ \alpha=\mathcal{E} .
\end{aligned}
$$

To see that $\mathcal{E}(A) \subset A^{\alpha}$ we compute:

$$
\begin{aligned}
\alpha \circ \mathcal{E} & =\alpha \circ\left(\iota_{A} \otimes \varphi\right) \circ \alpha \\
& =\left(\iota_{A} \otimes \iota_{H} \otimes \varphi\right) \circ\left(\alpha \otimes \iota_{H}\right) \circ \alpha \\
& =\left(\iota_{A} \otimes \iota_{H} \otimes \varphi\right) \circ\left(\iota_{A} \otimes \delta\right) \circ \alpha \\
& =\left(\iota_{A} \otimes(\underbrace{\left(\iota_{H} \otimes \varphi\right) \circ \delta}_{=\varphi(\cdot) \cdot 1_{H} \otimes 1_{\mathbb{C}}})\right) \circ \alpha \\
& =\left(\iota_{A} \otimes\left(\varphi(\cdot) \cdot 1_{H}\right)\right) \circ \alpha=\mathcal{E} \otimes 1_{H} .
\end{aligned}
$$

The rest is now clear.

Lemma 4. Let $B, A$ and $H$ be $C^{*}$-algebras. The identity map on $B \odot A \odot H$ extends to a homomorphism

$$
\rho: B \otimes_{\max }\left(A \otimes_{\min } H\right) \rightarrow\left(B \otimes_{\max } A\right) \otimes_{\min } H
$$


Proof. Let $B$ and $A$ act faithfully on a Hilbert space $\mathcal{H}$ in such a way that the $C^{*}$-algebra generated by $B$ and $A$ is $B \otimes_{\max } A$ and let $H$ act faithfully on a Hilbert space $\mathcal{K}$. The $C^{*}$-algebra generated by $B, A$ and $H$ on $\mathcal{H} \otimes \mathcal{K}$ is clearly $\left(B \otimes_{\max } A\right) \otimes_{\min } H$ and the $C^{*}$-algebra generated by $A$ and $H$ is $A \otimes_{\min } H$. Thus $\left(B \otimes_{\max } A\right) \otimes_{\min } H$ contains commuting copies of $B$ and $A \otimes_{\min } H$. By the universal property of $\otimes_{\max }$, we have a natural homomorphism $\rho$ : $B \otimes_{\max }\left(A \otimes_{\min } H\right) \rightarrow\left(B \otimes_{\max } A\right) \otimes_{\min } H$.

Let $\alpha: A \rightarrow A \otimes H$ be a coaction as above and $B$ a $C^{*}$-algebra. By the universality property of $\otimes_{\max }$, the map $\iota_{B} \odot \alpha: B \odot A \rightarrow B \odot(A \otimes H)$ extends to a homomorphism $\iota_{B} \otimes \alpha: B \otimes_{\max } A \rightarrow B \otimes_{\max }(A \otimes H)$. By composing it with the map $\rho$ in Lemma $\Theta$, we get a homomorphism

$$
\tilde{\alpha} \equiv \rho \circ\left(\iota_{B} \otimes \alpha\right): B \otimes_{\max } A \rightarrow\left(B \otimes_{\max } A\right) \otimes H .
$$

Lemma 5. $\tilde{\alpha}$ is a coaction of $H$ on $B \otimes_{\max } A$.

Proof. We first check the "counit" condition

$$
\left(\iota_{B \otimes \max } A \in\right) \circ \tilde{\alpha}=\iota_{B \otimes \max } A .
$$

Clearly we have

$\left(\left(\iota_{B \otimes_{\max } A} \otimes \epsilon\right) \circ \tilde{\alpha}\right)\left(b \otimes 1_{A}\right)=\left(\iota_{B \otimes_{\max } A} \otimes \epsilon\right)\left(b \otimes 1_{A} \otimes 1_{H}\right)=b \otimes 1_{A}, \quad b \in B$

Note that $\left(\iota_{B \otimes \max A} \otimes \epsilon\right) \circ \rho$ is the bounded map from $B \otimes_{\max }(A \otimes H)$ to $B \otimes_{\max } A, b \otimes a \otimes h \mapsto b \otimes(\epsilon(h) \cdot a), a \in A, b \in B, h \in H$, thus

$$
\left(\iota_{B \otimes \max } A \otimes \epsilon\right) \circ \rho: b \otimes x \mapsto b \otimes\left(\left(\iota_{A} \otimes \epsilon\right)(x)\right), \quad b \in B, x \in A \otimes H .
$$

By the counit property of $\epsilon$, we then have

$$
\begin{aligned}
\left(\left(\iota_{B \otimes_{\max } A} \otimes \epsilon\right) \circ \tilde{\alpha}\right)\left(1_{B} \otimes a\right) & =\left(\left(\iota_{B \otimes \max } A \theta\right) \circ \rho\right)\left(1_{B} \otimes \alpha(a)\right) \\
& =1_{B} \otimes\left(\left(\iota_{A} \otimes \epsilon\right) \alpha(a)\right)=1_{B} \otimes a, \quad a \in A .
\end{aligned}
$$

As $\epsilon$ is multiplicative $\left(\iota_{B \otimes \max } A\right) \otimes \epsilon \circ \tilde{\alpha}$ acts identically on $B \odot A$, hence on $B \otimes_{\max } A$ by continuity. As a consequence, $\tilde{\alpha}$ is injective. 
Since $\tilde{\alpha}$ is injective, the restriction of $\rho$ to $\left(\iota_{B} \otimes \alpha\right)\left(B \otimes_{\max } A\right)$ is injective too and we can define the following commuting diagrams

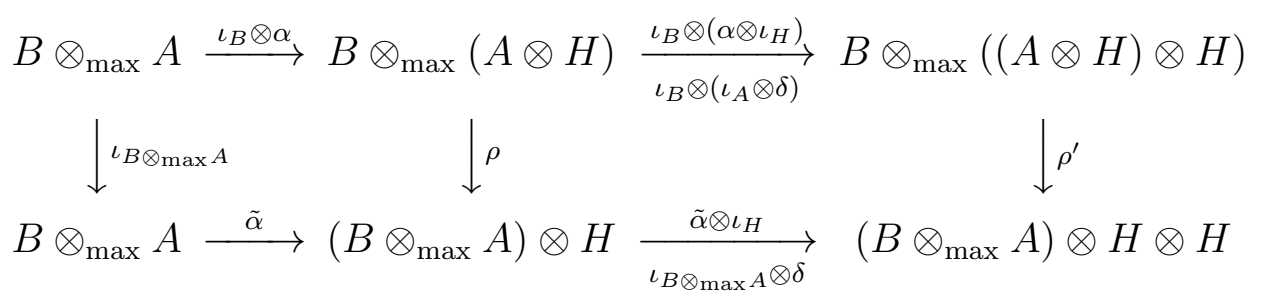

where $\rho^{\prime}$ is the natural map constructed by Lemma 4 , showing that $\tilde{\alpha}$ is a coaction.

Proposition 6. $\mathcal{E}$ is stably faithful.

Proof. Let $B$ a $C^{*}$-algebra. The conditional expectation $\tilde{\varepsilon}$ on $B \otimes_{\max } A$ extending $\iota_{B} \otimes \mathcal{E}$ on $B \odot A$ is the one associated with the coaction $\tilde{\alpha}$, hence it is faithful by Lemma 3 .

Corollary 7. Let $\alpha$ be a coaction of a compact quantum group $H$ with faithful Haar state and bounded counit on a $C^{*}$-algebra $A$. Then $A$ is nuclear iff $A^{\alpha}$ is nuclear.

Proof. This is a consequence of Propositions 11 and 6 .

Corollary 8. A compact quantum group $H$ with faithful Haar state and bounded counit is a nuclear $C^{*}$-algebra.

Proof. The comultiplication $\delta$ is a coaction of $H$ on itself and the statement follows by the above corollary, taking (4) into account. 


\section{Compact Group Actions: the $W^{*}$-case}

Before giving a $W^{*}$-version of Proposition 2, we need some preliminaries.

Let $X$ be a Banach space, and $Y$ a weak* dense Banach subspace of $X^{*}$. By a $\sigma(X, Y)$-continuous group of isometries $V: G \rightarrow B(X)$ we mean a $\sigma(X, Y)$-continuous homomorphism of the group $G$ into the group of all $\sigma(X, Y)$-continuous linear isometries of $X$. We note that to check the $\sigma(X, Y)$-continuity of the map $g \in G \rightarrow V_{g} \in B(X)$ we may verify that the maps $g \in G \rightarrow y\left(V_{g} x\right)$ are continuous when $x$ varies in a norm dense subset of $X$ and $y$ varies in a norm dense subset of $Y$.

Let $M$ and $N$ be von Neumann algebras. The binormal tensor product $N \otimes_{\text {bin }} M$ is the norm completion of $N \odot M$ with respect to the norm

$$
\|x\|=\sup _{\pi}\|\pi(x)\| \quad, \quad x \in N \odot M
$$

where the supremum ranges over all representations of $N \odot M$ with normal restrictions to $N \otimes 1_{M}$ and $1_{N} \otimes M$, called binormal representations [7].

Let $F \subset\left(N \otimes_{\text {bin }} M\right)^{*}$ be the Banach space of normal linear functionals associated with all such binormal representations.

Lemma 9. The kernel $J$ of the natural homomorphism

$$
\pi: N \otimes_{\text {bin }} M \rightarrow N \otimes_{\min } M
$$

is $\sigma\left(N \otimes_{\text {bin }} M, F\right)-$ closed.

Proof. When $M$ and $N$ act on $L^{2}(M)$ and $L^{2}(N)$ (respectively), the $C^{*}$ subalgebra of $B\left(L^{2}(N) \bar{\otimes} L^{2}(M)\right)$ generated by $N \otimes 1_{M}$ and $1_{N} \otimes M$ carries the minimal tensor product norm $\|\cdot\|_{\min }$. Thus we may identify it with $N \otimes_{\min } M$.

With this identification, $\pi$ is a binormal representation of $N \otimes_{\text {bin }} M$ on $L^{2}(N) \bar{\otimes} L^{2}(M)$, whose kernel is $J$. Note now that $\pi$ is continuous from the $\sigma\left(N \otimes_{\text {bin }} M, F\right)$-topology to the $\sigma$-weak topology of $B\left(L^{2}(N) \otimes L^{2}(M)\right)$, therefore $J$ is $\sigma\left(N \otimes_{\text {bin }} M, F\right)$-closed.

Proposition 10. Let $\alpha: G \rightarrow \operatorname{Aut}(M)$ be an action of a compact group $G$ on a von Neumann algebra $M$. Then $M$ is injective iff the fixed point subalgebra $M^{\alpha}$ is injective. 
Proof. If $M$ is injective, so is $M^{\alpha}$ because there is a conditional expectation from $M$ to $M^{\alpha}$.

Now assume that $M^{\alpha}$ is injective. In analogy with the proof of Proposition 1, we shall prove that, given any von Neumann algebra $N$, the ideal $J$ in Lemma 9 is $\{0\}$, what means that $M$ is semidiscrete, that is injective [3, 5].

The action $\beta=\iota \otimes \alpha$ on $N \odot M$ preserves the norm $\|\cdot\|_{\text {bin }}$, so each $\beta_{g}$ extends to a $*$-automorphism of $N \otimes_{\text {bin }} M$, still denoted by $\beta_{g}$. Furthermore, the map $G \ni g \mapsto \beta_{g}(x)$ is $\sigma(N \odot M, F)$-continuous for every $x \in N \odot M$. Therefore the action $\beta$ on $N \otimes_{\text {bin }} M$ is $\sigma\left(N \otimes_{\text {bin }} M, F\right)$-continuous.

Let us consider the conditional expectation $\mathcal{E}_{\alpha}: M \rightarrow M^{\alpha}$ defined by

$$
\mathcal{E}_{\alpha}(x)=\int \alpha_{g}(x) \mathrm{d} g, \quad x \in M
$$

The completely positive map $\iota \otimes \mathcal{E}_{\alpha}: N \otimes_{\min } M \rightarrow N \otimes_{\min } M$ has norm 1 , so

$$
\left\|\left(\iota \otimes \mathcal{E}_{\alpha}\right)(x)\right\|_{\min } \leq\|x\|_{\min } \leq\|x\|_{\text {bin }}, \quad x \in N \odot M .
$$

Now let $x \in N \odot M$. Then $\left(\iota \otimes \mathcal{E}_{\alpha}\right)(x) \in N \odot M^{\alpha}$. As $M^{\alpha}$ is semidiscrete, $\|\cdot\|_{\min }$ and $\|\cdot\|_{\text {bin }}$ coincide on $N \odot M^{\alpha}$. Therefore

$$
\left\|\left(\iota \otimes \mathcal{E}_{\alpha}\right)(x)\right\|_{\text {bin }} \leq\|x\|_{\text {bin }}, \quad x \in N \odot M,
$$

so $\left.\left(\iota \otimes \mathcal{E}_{\alpha}\right)\right|_{N \odot M}$ extends to a linear map of norm 1 from $N \otimes_{\text {bin }} M$ to itself, still denoted by $\iota \otimes \mathcal{E}_{\alpha}$, whose range is contained in the closure of $N \odot M^{\alpha}$ in $N \otimes_{\text {bin }} M$.

Let $x \in N \otimes_{\text {bin }} M$ and choose $x_{n} \in N \odot M, n \geq 1$, with $\left\|x-x_{n}\right\|_{\text {bin }} \rightarrow 0$. Then, for all $\varphi \in F$,

$$
\begin{aligned}
\varphi\left(\left(\iota_{N} \otimes \mathcal{E}_{\alpha}\right)(x)\right)=\lim _{n} \varphi\left(\left(\iota_{N} \otimes \mathcal{E}_{\alpha}\right)\left(x_{n}\right)\right) \\
=\lim _{n} \int \varphi\left(\beta_{g}\left(x_{n}\right)\right) \mathrm{d} g=\int \varphi\left(\beta_{g}(x)\right) \mathrm{d} g,
\end{aligned}
$$

SO

$$
\mathcal{E}_{\beta}(x)=\int \beta_{g}(x) \mathrm{d} g
$$

exists in the $\sigma\left(N \otimes_{\text {bin }} M, F\right)$-weak sense and $\mathcal{E}_{\beta}(x)=\left(\iota_{N} \otimes \mathcal{E}_{\alpha}\right)(x)$ for all $x \in N \odot M$. In particular, the range of $\mathcal{E}_{\beta}$ is contained in the closure of $N \odot M^{\alpha}$. Moreover $\mathcal{E}_{\beta}$ is faithful. 
Now let $x \in J$ be a positive element. Since each $\beta_{g}$ leaves $J$ globally invariant, $\beta_{g}(x) \in J$ for all $g \in G$. By the Hahn-Banach theorem $\mathcal{E}_{\beta}(x)$ belongs to the $\sigma\left(N \otimes_{\text {bin }} M, F\right)$-closed linear span of $\left\{\beta_{g}(x) ; g \in G\right\}$, thus $\mathcal{E}_{\beta}(x) \in J$ by Lemma 9 .

But $\mathcal{E}_{\beta}(x)$ is also in the closure of $N \odot M^{\alpha}$ which, by the semidiscreteness of $M^{\alpha}$, intersects $J$ only in 0 . Consequently $\mathcal{E}_{\beta}(x)=0$ and the faithfulness of $\mathcal{E}_{\beta}$ yields $x=0$. We conclude that $J$ is trivial and $M$ is semidiscrete, thus injective.

Acknowledgements. We thank S. Woronowicz for a clarifying answer to a question and F. P. Boca for calling our attention to reference [2].

\section{References}

[1] Baaj, P. S., Skandalis, G., Unitaires multiplicatifs et dualité pour les produits croisés des $C^{*}$-algèbres, Ann. Scient. Ec. Norm, Sup. $4^{e}$ série 26 (1993), 425-488.

[2] Boca, F. P., Ergodic actions of compact matrix pseudogroups on $C^{*}$-algebras, in "Recent Advances in Operator Algebras", Astérisque 232 (1995), 93-109.

[3] Choi, M. D., Effros, E., Nuclear $C^{*}$-algebras and injectivity; the general case, Indiana Univ. Math. J. 26 (1977), 443-446.

[4] Connes, A., Classification of injective factors, Ann. Math. 104 (1976), 73-115.

[5] Connes, A., On the equivalence between injectivity and semidiscreteness for operator algebras, in "Algèbres d'Opérateurs et leurs Applications en Physique Mathématique" D. Kastler ed., Colloques Internationaux du CNRS 274 (1979) 107-112

[6] Doplicher, S., Roberts, J.E., Duals of compact Lie groups realized in the Cuntz algebras and their actions on $C^{*}$-algebras, J. Funct. Anal. 74 (1987), 96-120.

[7] Effros, E., Lance, C., Tensor product of operator algebras, Adv. Math. 25 (1977), $1-34$.

[8] Høegh-Krohn, R., Landstad, M. B., Størmer, E., Compact ergodic groups of automorphisms, Ann. Math. 114 (1981), 75-86.

[9] Kustermans, J., Vaes, S., The operator algebra approach to quantum groups, Proc. Natl. Acad. Sci. 97 (2000), no. 2, 547-552.

[10] Masuda, T., Nakagami, Y., A von Neumann algebra framework for the duality of the quantum groups, Publ. Res. Inst. Math. Sci. 30 (1994), no. 5, 799-850.

[11] Strătilă, Ş., Voiculescu, D. V., Zsidó, L. On crossed products I, Revue Roum. Math. Pures et Appl. 21 (1976), 1411-1449 
[12] Van Daele, A., The Haar measure on a compact quantum pseudogroup, Proc. Amer. Math. Soc. 123 (1995), 3125-3128.

[13] Van Daele, A., Wang, S. Universal quantum groups, Int. J. Math. 72 (1996), 255-264.

[14] Wang, S., Ergodic actions of universal quantum groups on operator algebras, Commun. Math. Phys. 203 (1999), 481-498.

[15] Woronowicz, S., Compact matrix pseudogroups, Commun. Math. Phys. 111 (1987), 613-665.

[16] Woronowicz, S., Compact quantum groups, in "Symétries Quantiques", A. Connes, K. Gawedzki, J. Zinn-Justin eds., Les Houches, Session LXIV (1 Août - 8 Sept. 1995), Elsevier Science, 1998. 